\title{
Carga de enfermedad de sepsis en el lactante menor. Un gran desafío multidisciplinario para Latinoamérica
}

\author{
The burden of sepsis in infants: a big multidisciplinary challenge \\ for Latin-America
}

\section{Franco Díaz Rubio}

aUnidad de Paciente Crítico Pediátrico, Hospital El Carmen de Maipú. Santiago, Chile

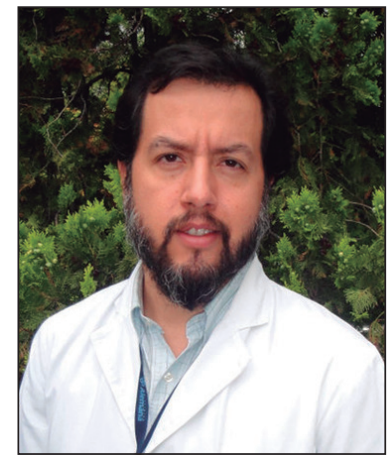

Las infecciones graves y sepsis han sido identificadas como uno de los grandes problemas de salud pública en el mundo ${ }^{1}$. Estudios de prevalencia en los últimos años, han registrado información más detallada de países de bajos y medianos ingresos, dejando en evidencia un problema más profundo de lo que pensábamos, duplicando la carga de enfermedad de sepsis en el mundo ${ }^{2}$. La carga de enfermedad es una forma de cuantificar el impacto de un problema de salud, y recientemente el grupo The Global Burden of Disease (www.healthdata.org) ha logrado determinar la carga de enfermedad de más de 100 enfermedades a nivel mundial reportando su incidencia. Con estos datos, Rudd KE et al. estimaron una incidencia global de sepsis de 677,5 (535,7-876,1) casos por 100.000 habitantes, siendo responsable de aproximadamente la mitad de las muertes en el mundo; sin embargo, existen grandes diferencias geográficas según ingreso per cápita, desarrollo sociodemográfico y también según edad ${ }^{2}$. Es así como la incidencia ajustada más alta ocurre en menores de 5 años, en especial en lactantes menores ${ }^{2}$. En este grupo etario se observa la mayor mortalidad por sepsis, siendo atribuida como causa de muerte en el $75 \%$ de los fallecidos, superando con creces las enfermedades no transmisibles y politraumatismos ${ }^{2}$. Otros estudios globales pediátricos han reportado que sepsis en menores de 1 año tiene una mortalidad cercana al $40 \%$ en niños hospitalizados en unidades de cuidados intensivos pediátricos (UCIPs) ${ }^{3}$. Reportes latinoamericanos confirman estos datos (tabla 1$)^{4-6}$, revelando que las infecciones graves en el lactante menor son la principal causa de mortalidad infantil prevenible en el continente. Latinoamérica es una región heterogénea geopolíticamente y también en su desarrollo económico. Al igual que los datos mundiales, existe una relación inversa entre desarrollo sociodemográfico y mortalidad. Este alcance no solo responde a la distribución política/económica, sino también a grandes diferencias dentro de los países. Esto fue recientemente descrito por González $\mathrm{F}$ et al. ${ }^{7}$ en una de las áreas urbanas más grande de Latinoamérica, la Región Metropolitana de Santiago de Chile, en que la mortalidad infantil es hasta cuatro veces mas alta al comparar distintas comunas. Jabornisky R. et al., en un análisis de 45 unidades de cuidados intensivos pediátricos de Argentina, encontró que los pacientes ingresados por sepsis desde zonas rurales tenían un riesgo de muerte 3,4 veces mayor que los que procedían de localidades urbanas ${ }^{6}$.

En dos artículos recientes de Revista Chilena de Pediatría, Donoso A et al. ${ }^{8}$ y Moya $\mathrm{M}$ et al. ${ }^{9}$, abordan

Correspondencia:

Franco Díaz Rubio

francodiazr@gmail.com 
Tabla 1. Estudios Latinoamericanos de frecuencia y mortalidad de sepsis en pacientes ingresados a unidades de cuidados intensivos pediátricos

\begin{tabular}{|c|c|c|c|c|}
\hline Autores & País & Año(s) de registro & Frecuencia & Mortalidad \\
\hline Jabornisky et al. & Argentina & 2008 & $13,8 \%$ & $31,60 \%$ \\
\hline Ribeiro et al. & Brasil & 1993-1994 & N/A & $56,10 \%$ \\
\hline Carvalho et al. & Brasil & $1999-2000$ & $44,5 \%$ & $14,90 \%$ \\
\hline Arriagada et al. & Chile & $2006-2007$ & $3,5 \%$ & $19 \%$ \\
\hline Bustos et al.* & Chile & $2009-2011$ & N/A & $13,5 \%$ \\
\hline Jaramillo-Bustamante et al. & Colombia & $2009-2010$ & N/A & $18 \%$ \\
\hline Rovira et al. & Cuba & $2008-2009$ & N/A & $12,40 \%$ \\
\hline Ponce K. et al. ** & Honduras & 2006 & N/A & $24,5 \%$ \\
\hline Corrales et al. & México & $2013-2014$ & N/A & $38 \%$ \\
\hline Tapia-Cabrera et al. & México & 2016 & N/A & $28,50 \%$ \\
\hline Salcedo-Reyes et al. & México & $2005-2008$ & $43,9 \%$ & $46,88 \%$ \\
\hline Manzanares et al. & Nicaragua & 2016 & $79 \%$ & $13,8 \%$ \\
\hline Sáez-LLorens et al. & Panamá & 1981-1992 & $18 \%$ & $39 \%$ \\
\hline Alonso et al. & Paraguay & 2010 & $57,2 \%$ & $18,3 \% \%$ \\
\hline Tantealán et al. & Perú & 1996-1997 & $46 \%$ & $37 \%$ \\
\hline Curiel et al. & Venezuela & $2007-2008$ & $20 \%$ & $17,5 \%$ \\
\hline SPROUT*** & Sudamérica & 2013-2014 & $16,3 \%$ & $11 \%$ \\
\hline
\end{tabular}

*Menores de 5 años, ** menores de 1 año, *** unidades de cuidados intensivos pediátricos participantes en SPROUT ${ }^{3}$.

las infecciones graves en niños desde 2 puntos de vista. El primero corresponde a una perspectiva histórica de tratamiento de shock séptico (en este caso específicamente meningocóccico) durante el último siglo. En los albores de la pediatría chilena y la comprensión de la fisiopatológica de sepsis, los autores realizan una descripción del contexto sociocultural de chile, su transición epidemiológica y los distintos tratamientos intentados para esta infección grave. Los autores explican en detalle las contribuciones de Drs. Eduardo Cruz-Coke, Luis Calvo Mackenna y Salvador Allende, entre otros, en el desarrollo de una aproximación científica a las enfermedades de los niños y la articulación del sistema de salud con énfasis en la pediatría, que ha llevado a reducir la una mortalidad infantil de 231/1.000 nacidos vivos en 1930 a 6/1.000 nacidos vivos en $2016^{1}$.

Moya $\mathrm{M}$ et al. ${ }^{7}$, analizan una cohorte prospectiva de niños menores de 3 meses de vida, hospitalizados por síndrome febril sin foco en 2 hospitales pediátricos públicos de Santiago de Chile. El diagnóstico de egreso fue una infección bacteriana seria (IBS) en aproximadamente un tercio de los pacientes, siendo las infecciones del tracto urinario, bacteremia y onfalitis los focos más frecuentes. En esta cohorte los autores no encontraron diferencias clínicas ni de laboratorio significativas entre IBS y aquellos niños cuyo diagnóstico de egreso no fue IBS. Este estudio se suma a otras series publicadas en la región, cuyos resultados son heterogéneos dependiendo de la proporción de pacientes menores a un mes, puntaje de evaluación estandarizada, uso de exámenes complementarios (i.e. procalcitonina) y características de los centros participantes (i.e. pediátricos vs generales; público $v s$ privado; $)^{10-13}$. La importancia del estudio de Moya $\mathrm{M}$ et al. ${ }^{9}$ radica en la descripción clínica y demográfica de un grupo de lactantes menores con un alto riesgo de IBS, sepsis y mortalidad asociada. Dentro de los resultados, es llamativo que un porcentaje menor al $10 \%$ requirió atención en cuidados intensivos, la mayoría de los pacientes recibió terapia antibiótica y no hubo fallecidos. Las características propias de la población atendida en los centros estudiados probablemente son factores que inciden en la baja mortalidad y gravedad encontrada: carácter urbano, buena accesibilidad a proveedores de salud, atención en un hospital de especialidad pediátrica, entre otros, y de un país clasificado de altos ingresos ${ }^{1}$. Sin embargo, esta no es la realidad de muchos lugares de Chile y en Latinoamérica. Es necesario hacer hincapié en la potencial gravedad y morbimortalidad asociadas a un diagnóstico errado o tardío de IBS en el lactante menor. Moya $\mathrm{M}$ et al. demuestran que, en menores de 3 meses hospitalizados por síndrome febril sin foco, ninguno de los exámenes realizados en forma inicial fueron predictores de IBS. 
Los dos artículos mencionados nos recuerdan el camino difícil que hemos recorrido para mejorar el tratamiento de las infecciones graves en niños, y también los desafíos que quedan, especialmente para definir grupos de riesgo de morbimortalidad por infecciones graves. Nuevos biomarcadores están en estudio para abordar este problema, pero más que un examen único con un punto de corte y resultado binario, probablemente requerirá una evaluación de vías moleculares relacionadas con inflamación e infección, así como las características del agente infeccioso y la predisposición particular del paciente.

\section{Conflicto de intereses}

El autor declara no tener conflicto de intereses.

\section{Referencias}

1. United Nations Interagency Group for Child Mortality Estimation (UN IGME). Levels \& Trends in Child Mortality: Report 2018. Estimates developed by the United Nations Inter-agency Group for Child Mortality Estimation', United Nations Children's Fund, New York, 2018. Accedido on-line 14 de Febrero 2020 [http://scalingupnutrition.org/wpcontent/uploads/2018/09/ChildMortalityReport-2018.pdf]

2. Rudd KE, Johnson SC, Agesa KM, et al. Global, regional, and national sepsis incidence and mortality, 1990-2017: analysis for the Global Burden of Disease Study. Lancet. 2020;395(10219):200-211.

3. Weiss SL, Fitzgerald JC, Pappachan J, et al; Sepsis Prevalence, Outcomes, and Therapies (SPROUT) Study Investigators and Pediatric Acute Lung Injury and Sepsis Investigators (PALISI) Network. Global epidemiology of pediatric severe sepsis: the sepsis prevalence, outcomes, and therapies study. Am J Respir Crit Care Med. 2015;191(10):1147-57.

4. de Souza DC, Shieh HH, Barreira ER,
Ventura AM, Bousso A, Troster EJ; LAPSES Group. Epidemiology of Sepsis in Children Admitted to PICUs in South America. Pediatr Crit Care Med. 2016;17(8):727-34.

5. Jaramillo-Bustamante JC, Marín-Agudelo A, Fernández-Laverde M, Bareño-Silva J. Epidemiología de la sepsis en pediatría: primer estudio colombiano multicéntrico. CES Medicina 2009;23(1):85-92.

6. Jabornisky R, Sáenz SS, Capocasa P, et al. Epidemiological study of pediatric severe sepsis in Argentina. Arch Argent Pediatr. 2019;117 Supl 3:S135-S156

7. González F, Alfaro T, Horna-Campos O. Mortalidad Infantil en las comunas de la Región Metropolitana, periodo 20052014. Rev Chil Pediatr. 2020;91(1): 34-45.

8. Donoso FA, Arriagada SD. A propósito de la epidemia meningocóccica chilena (1941-1942): El niño con shock séptico hace 80 años desde la perspectiva médica y social. Rev Chil Pediatr Forthcoming 2020;91(3). Disponible en: DOI:10.32641/ rchped.v91i3.1714 [Accessed $17 \mathrm{abr}$. 2020].

9. Moya M, Couble B, Piñera C, el al. Utilidad de los parámetros clínicos y de laboratorio básicos para predecir infección bacteriana seria en menores de 3 meses que se hospitalizan por síndrome febril sin foco. Rev Chil Pediatr. 2020;91(2).

10. Brockmann P, Ibarra X, Silva I, Hirsch T. Etiología del síndrome febril agudo sin foco en niños bajo 36 meses de edad que consultan a un servicio de urgencia. Rev Chilena Infectol. 2007;24(1):33-9.

11. Torregrosa C, García C, Sciarotta J, Vay C, Caíno S, Ellis A. Factores asociados a riesgo de infección bacteriana grave en niños bajo 24 meses de edad, internados por fiebre sin foco aparente. Rev Chilena Infectol. 2010;27(4):308-14.

12. Vásquez-Hoyos P, Soto F, Pinzón D, González D, Peña C. Caracterización de pacientes pediátricos con hemocultivos positivos del servicio de cuidado intensivo pediátrico del Hospital San José Bogotá, abril 2012 a 2017. Infectio 2019;23(2):1838.

13. Barra X, Viviani T, Peña AM, Cerda J. Síndrome febril sin foco y sospecha de infección bacteriana en niños entre 6 semanas y 36 meses. Rev Chil Pediatr. 2008;79(4):388-92. 